\title{
TAVI patient selection and multidisciplinary evaluation
}

\section{Editorial}

Calcific aortic stenosis (AS) is the most common manifestation of valvular heart disease. Aortic valve replacement (AVR) is the only effective treatment for symptomatic AS, being surgery the preferred AVR option for patients across all age groups. Although surgical AVR has an overall low operative mortality, ${ }^{1}$ elderly and frail patients are at a much higher risk, presumably owing to the presence of multiple coexisting conditions. ${ }^{2}$ Cribier et al., ${ }^{3}$ performed the first human transcatheter aortic valve implantation (TAVI) in 2002, a procedure typically targeted at patients with severe AS who were unfit for conventional surgery. ${ }^{4-6}$ Since then, the number of patients treated with TAVI has grown exponentially, i. e., more than 70000 valves have been implanted in 2015 and this number is likely to quadruple to over 280000 by $2025 .{ }^{7}$ Recent studies, including some of our group, have shown favorable outcomes of TAVI in different clinical scenarios..$^{8-11}$ TAVI is now considered by some physicians as the standard of care for these patients and is also a reasonable alternative to surgery in high-risk operable patients.

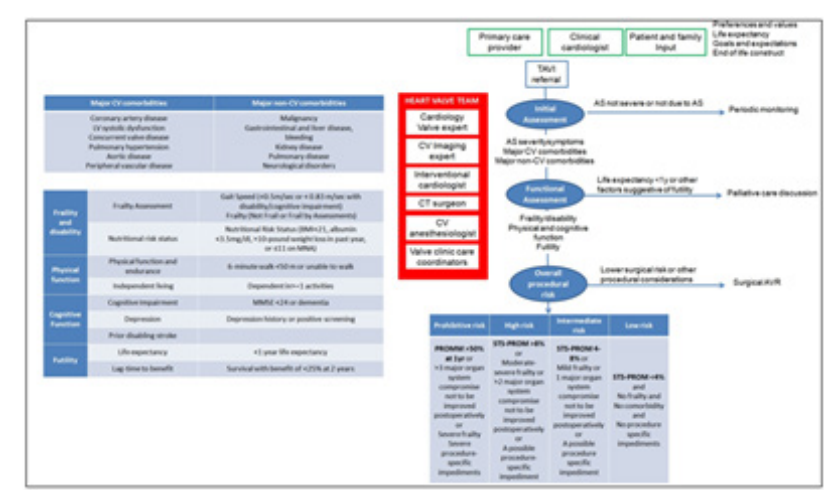

Figure I TAVI patient selection and multidisciplinary evaluation.

TAVI:Transcatheter Aortic Valve Implantation;AS:Aortic Stenosis; AVR:Aortic Valve Replacement; BMI: Body Mass Index; CV: Cardiovascular; LV: Left Ventricular; MNA: Mini Nutritional Assessment; MMSE: Mini Mental State Examination; CT: Cardiothoracic; PROMM: Predicted Risk of Mortality or Major Morbidity; STS-PROM: Predicted Risk of Mortality.

Selection of TAVI candidates should ideally be made by a 'heart team', with a particular expertise in valvular heart disease, including interventional cardiologists, cardiac and vascular surgeons, cardiovascular anesthesiologists, imaging specialists and specialized cardiovascular nursing. ${ }^{12}$ The "heart team" approach is based on a comprehensive understanding of the risk-benefit ratio of different treatment strategies and integration of patient preferences. As shown in Figure 1, when considering different treatment options, the "heart team" should consider the presence of cardiovascular and non-cardiovascular mayor comorbidities, flailty and disability, as well as physical and cognitive function. All these variables should be carefully considered to avoid futile interventions. This is a clear
Volume 8 Issue 3 - 2017

\author{
Amelia Carro,' Isaac Pascual,, ${ }^{2}$ Cesar Moris, ${ }^{2}$ \\ Pablo Avanzas ${ }^{2}$ \\ 'Department of Cardiology, Instituto Corvilud, Spain \\ ${ }^{2}$ Cardiac Catheterization Laboratories, Area del Coraz
}

Correspondence: Pablo Avanzas Fernandez, Cardiac Catheterization Laboratories, Area del Corazón, Hospital Universitario Central de Asturias, Oviedo, Spain, Email avanzas@secardiologia.es

Received: February 14, 2017| Published: March 03, 2017

example of a shared decision-making process, that involves education of the patient, their family, and the referring physician about treatment alternatives. Patient goals and expectations should be established early in this process in the context of a discussion of life expectancy, anticipated improvement in symptoms or survival, and end-of-life constructs, when appropriate. At this point, the overall procedural risk should be calculated. Several cardiac surgical risk algorithms have been developed over the last 20years. Despite their known limitations, the logistic EuroSCORE and the STS (Society of Thoracic Surgeons) Predicted Risk of Mortality score have been used to define "high surgical risk" patients in TAVI trials. The STS-PROM risk calculator is a widely accepted tool, with classification into 3 initial categories of risk based on the STS score: $<4 \%$ (low risk), $4-8 \%$ (intermediate risk), and $>8 \%$ (high risk). Clinical judgment should play an integral role in the selection of patients for TAVI. Thus, risk scores should not dictate but guide clinical decision making.

\section{Conclusion}

TAVI is currently reserved for patients with severe aortic stenosis deemed high or prohibitive surgical risk-but this may change in the future. Identification of high or prohibitive surgical risk patient should rely on the clinical judgment of a Heart Team (specifically, interventional cardiologists and cardiac surgeons) in conjunction with information gleaned from patient and family inputs and surgical risk scores such as the STS Predicted Risk of Mortality.

\section{Acknowledgments}

None.

\section{Conflicts of interest}

Author declares there is no conflicts opf interest. 


\section{Funding}

None.

\section{References}

1. Edwards FH, Peterson ED, Coombs LP, et al. Prediction of operative mortality after valve replacement surgery. $J$ Am Coll Cardiol. 2011;37(3):885-892.

2. Iribarne A, Easterwood R, Chan EY, et al. The golden age of minimally invasive cardiothoracic surgery: current and future perspectives. Future Cardiol . 2011;7(3):333-346.

3. Cribier A, Eltchaninoff H, Bash A, et al. Percutaneous transcatheter implantation of an aortic valve prosthesis for calcific aortic stenosis: first human case description. Circulation. 2002; 106(24):3006-3008.

4. Leon MB, Smith CR, Mack M, et al. Transcatheter aortic-valve implantation for aortic stenosis in patients who cannot undergo surgery. N Engl J Med. 2010;363(17):1597-1607.

5. Adams DH, Popma JJ, Reardon MJ. Transcatheter aortic-valve replacement with a self-expanding prosthesis. $N$ Engl J Med. 2014;371(10):967-968.

6. Popma JJ, Adams DH, Reardon MJ, et al. Transcatheter aortic valve replacement using a self-expanding bioprosthesis in patients with severe aortic stenosis at extreme risk for surgery. J Am Coll Cardiol. 2014;63(19):1972-1981.
7. Morís C, Pascual I, Avanzas P. Will TAVI Be the Standard of Care in the Treatment of Aortic Stenosis?. Rev Esp Cardiol (Engl Ed) . 2016;69(12):1131-1134.

8. Del Valle R, Pascual I, Silva J, et al. Transapical Implantation in the Catheterization Laboratory of the Second Generation Engager Aortic Valve. Rev Esp Cardiol (Engl Ed) . 2016;69(4):451-453.

9. Pascual I, Muñoz-García AJ, López-Otero D, et al. Transcatheter aortic valve implantation in very elderly patients: immediate results and medium term follow-up. J Geriatr Cardiol. 2015;12(4):340-345.

10. Muñoz-García AJ, Pascual I, Avanzas P, et al. New Generation CoreValve Evolut $^{\mathrm{TM}}$ R 23mm Aortic Valve Prosthesis: Initial Experience. Rev Esp Cardiol (Engl Ed). 2015;68(8):721-722.

11. Pascual I, Muñoz-García AJ, López-Otero D, et al. Long-term outcome of cirrhotic patients with severe aortic stenosis treated with transcatheter aortic valve implantation. Rev Esp Cardiol (Engl Ed. 2015;68(4):353-354.

12. Vahanian A, Alfieri O, Andreotti F, et al. Guidelines on the management of valvular heart disease (version 2012). Joint Task Force on the Management of Valvular Heart Disease of the European Society of Cardiology (ESC); European Association for Cardio-Thoracic Surgery (EACTS). Eur Heart J. 2012;33(19):2451-2496. 